África (São Paulo, 1978, Online), São Paulo, n. 42, p. 77-85, 2021

http://dx.doi.org/

\title{
Nouveaux themes de recherche dans les litteratures africaines, defis et possibilites d'ecrire et d'enseigner ces litteratures dans les langues africaines
}

OLABIYI Yai ${ }^{\prime}$

Resumé: Malhereusement les institutions universitaires africaines ne correspondent pas aux besoins et aux intérêts de l'Afrique. Pendant cinquante ans et plus, nous avons "pensé avec la tête des autres" et avons échoué, en règle générale. Si, aujourd'hui, nous estimons que "l'heure de nous-mêmes a sonné" (Césaire), il nous faudra penser une nouvelle approche de l'Université, par nous-mêmes. Et plus particulièrement dans les sciences sociales et humaines, cette approche doit privilégier l'articulation de "l'influx" et de l' 'afflux".

Mot-clés: Universitè africaine ; Littératures africaines

Resumo: Infelizmente, as instituições acadêmicas africanas não correspondem às necessidades e interesses da África. Por cinquenta anos ou mais, "pensamos com a cabeça de outras pessoas" e falhamos, como regra. Se hoje sentimos que "chegou a hora para nós" (Césaire), teremos que pensar numa nova abordagem da Universidade, por nós mesmos. E mais particularmente nas ciências sociais e humanas, esta abordagem deve favorecer a articulação de "influxo" e "influxo".

Ambassadeur délégué permanent du Bénin auprès de l'UNESCO, Ancien Président du Conseil exécutif de l'UNESCO. 
OLABIYI YAI Nouveaux themes de recherche dans les litteratures africaines, defis et possibilites d'ecrire et d'enseigner ces litteratures dans les langues africaines

Palavras-chave: Universidade africana; Literaturas africanas

Lidée, à l'initiative du Brésil, d'organiser, ou, s'agissant du Brésil, d'orchestrer une réflexion bi continentale sur 'L'Université Africaine dans le contexte de la mondialisation" me ramène irrésistiblement à l'esprit les vers de deux de nos plus illustres ancêtres-poètes: d'abord le cubain Nicolás Guillén qui, dans son poème au titre significatif, "Llegada", nous rappelle:

Traemos nuestro rasgo

al perfil definitivo de America.

Ainsi donc le Brésil, grande terre de flux africain, persévère-t-il dans sa généreuse volonté d'associer l'Afrique dans le titanesque effort d'enfanter une nouvelle humanité à partir des douleurs d'hier et de l'expérience d'aujourd'hui sainement et communément assumées.

C'est ensuite Carlos Drummond de Andrade notre deuxième ancêtre-poète, dont il me plait d'invoquer la voix, dans son célèbre Canto brasileiro:

Brasa sem brasão brasil paixăo
de vida popular em mundo aberto
à confiança dos homens.
Assim me vejo e toco: brasileiro
sem limites traçados para o amor humano.

Je souhaite que ces deux poètes nous servent d'ancêtres tutélaires et que leurs vers nous inspirent tout au long de nos travaux.

C'est à dessein que, plus haut, j’ai utilisé le terme "persévérer", s'agissant de l'initiative brésilienne. Il est opportun en effet de rappeler qu'il y a un demi-siècle, le Brésil avait organisé, de concert avec l'UNESCO, un colloque de même nature mais de plus grande envergure parce qu'il était inaugural, pour "entamer un dialogue sur les anciens rapports de l'Afrique et de l'Amérique Latine et sur les perspectives qui souffrent à leur future collaboration" (Carneiro: 185). Paulo E. de Berrêdo Carneiro avait alors souhaité que ce colloque "puisse ... rester dans les annales de notre temps comme un 
África (São Paulo, 1978, Online), São Paulo, n. 42, p. 77-85, 2021

exemple de vraie communion politique et morale, un témoignage d'authentique fraternité". Reprenons le souhait à notre compte et faisons la promesse que nos assises donnent le "la" de la renaissance de l'Université Africaine.

Avant tout propos sur les littératures africaines et leur enseignement à l'Université, il me semble de bonne méthode de commencer par une réflexion, même sommaire, sur l'idée d'une université africaine. Les universités africaines dont nous nous donnons pour tâche de procéder à une évaluation après un demi-siècle d'indépendance sont, de l'avis unanime, un "héritage" colonial, euro-américain. C'est précisément ce concept d' 'héritage colonial", qui est à mon avis moins innocent qu'il n'y paraît, qu'il s' agit de critiquer radicalement si nous tenons à changer de paradigme. Car, du point de vue africain, il ne saurait y avait "héritage colonial" sauf à nous laisser piéger dès l'amont. En Afrique, on hérite de son père ou de sa mère. On n'hérite pas du parâtre ou de la marâtre imposés qu'est le système colonial dans son ensemble. Or l'axiome fondateur, quoique non explicite, qui a présidé à la création de ces institutions est qu'en matière d'université, l'Afrique est une table rase. Loin de constituer un quelconque "héritage", ces universités nous sont "données" ou "imposées" par ceux que j’ai appelé nos parâtres /marâtres.

Notre premier geste critique consistera donc à rechercher et retrouver nos traditions universitaires africaines les plus anciennes (Egypte pharaonique, Tombouctou [Sankoré] Ife, Zimbabwe etc.).

Pour emprunter une métaphone célèbre à Joseph $\mathrm{Ki}$-Zerbo, il nous faut REMEMBRER L'OSIRIS UNIVERSITAIRE AFRICAIN.

Connaître, critiquer et assumer ces traditions avec leur limon comme leurs scories et les harmoniser avec les autres traditions, l'Euro-américaine (JASPERS, 2008), l'indienne (Tagore), la chinoise, la japonaise et bien d'autres.

C'est là, à mon sens une tâche incontournable, un préalable à toute entreprise de renaissance africaine (une Inventio et Sinfonia pour emprunter le titre d'une composition de J.S. Bach).

Le présupposé de table rase universitaire africaine a pour corollaire que l'Occident a pensé "nos" universités pour nous, sans nous. Il n'est point étonnant dès lors que les institutions universitaires africaines ne correspondent pas aux besoins et aux intérêts de l'Afrique. Pendant cinquante ans et plus, nous avons "pensé avec la tête des autres" et avons échoué, en règle géné- 
OLABIYI YAI Nouveaux themes de recherche dans les litteratures africaines, defis et possibilites d'ecrire et d'enseigner ces litteratures dans les langues africaines

rale. Si, aujourd'hui, nous estimons que "l'heure de nous-mêmes a sonné" (Césaire), il nous faudra penser une nouvelle approche de l'Université, par nous-mêmes. Et plus particulièrement dans les sciences sociales et humaines, cette approche doit privilégier l'articulation de "l'influx" et de l' "afflux". Et les vers du poète ici se font vaticination.

La relance ici se fait par le vent qui d'Afrique vient la relance ici se fait par l'influx plus encore que par l'afflux

(Césaire: 41).

Pour caractériser le paysage littéraire du continent africain aujourd'hui, les qualificatifs suivants viennent à l'esprit et s'y bousculent, sans qu'aucun ne décrive cependant le phénomène de façon satisfaisante ou exhaustive : le paysage littéraire est riche, bigarré, foisonnant, divers, chaotique et en transition vers plusieurs inconnus. Le champ littéraire africain est complexe ; il est également le lieu de nombreux enjeux.

L'incontournable question-spectre de l'identité continue de hanter le continent: Qu'est-ce-que la Littérature africaine? Cette question, qui n'a de sens que chez nous, doigte notre condition coloniale, notre exception en la matière. Car il ne viendrait à l'esprit de personne de demander 'qu' est-ce que la littérature anglaise, espagnole, russe, française"? Où l'on voit que cette question est, en son essence, non pas littéraire, mais politique. La réponse n'en appartient donc pas aux seuls hommes et femmes de lettres, même si ceux-ci peuvent y contribuer. Il faut en prendre son parti, la solution durable au problème de l'identité de la littérature africaine relève du politique. Notre position en la matière est qu'il vaut mieux s'éloigner des approches manichéennes et s'inspirer en la matière des traditions intellectuelles africaines qui privilégient l'addition et non la substitution, qui encouragent à tisser la nouvelle corde au bout de la vieille, à articuler influx et afflux.

Sur la base de cette prémisse, nous appellerons Littérature africaine tout texte, oral ou écrit de caractère littéraire produit par un Africain, quelle que soit la langue dans laquelle il est exprimé. Cette définition provisoire, a l'avantage de reconnaître au politique son rôle d'élément déterminant. Elle repose surtout sur l'espoir que les citoyens africains amèneront leurs gouvernements à adopter des politiques de plurilinguisme à base africaine, susceptibles 
de former des Africains lettrés, multilingues, qui disposent ainsi de ce que l'on appelle aujourd'hui mais de façon fallacieuse le choix de la / des langue(s).

Cette définition a l'avantage également de ne pas nous empêtrer dans des discussions inhibitrices mais de partir de constats, de l'existant littéraire sur le continent, tout en n'interdisant pas des considérations liées aux valeurs, concept incontournable en littérature.

Notre continent peut s'enorgueillir de trois types de littérature:

1. Les littératures orales en langues autochtones africaines;

2. Les littératures écrites en langues autochtones africaines;

3. Les littératures écrites en langues européennes.

Cette dernière catégorie occupe une position hégémonique (public important et puissant en dehors du continent, prix prestigieux y compris le Nobel... etc.).

Nous nous intéressons dans le reste de cette communication aux défis et perspectives des littératures africaines exprimées en langues africaines.

La condition de possibilité première d'une littérature écrite en une langue africaine est l'utilisation courante dans la sphère publique, puis sur le plan esthétique de la langue concernée. Le premier défi auquel elle est confrontée est ainsi un défi existentiel, lié à la menace de mort qui pèse sur la plupart des langues du continent. Nous avons vu que ce défi est politique. De nombreuses études scientifiques existent aujourd'hui, qui peuvent servir de base d'appui pour tout gouvernement africain qui le désire de se donner une politique linguistique volontariste de multilinguisme. Des expérimentations ont également été menées qui démontrent qu'une telle politique n'est pas une vue de l'esprit. Une Académie Africaine des Langues (ACALAN) a même été créée par l'Union Africaine. Ce qui fait tragiquement défaut, c'est la volonté politique des états, un ingrédient essentiel pourtant. A défaut de celle-ci, des initiatives se prennent çà et là par les communautés ou des membres de la société civile, pour faire naître, pratiquement ex nihilo, ce type de littérature (NGUGI, 1993, DIOP, Boubacar Boris, 2011). Ces initiatives quelque significatives et louables qu'elles sont pour la naissance de ce que l'on peut appeler les littératures en langues africaines de deuxième géné- 
OLABIYI YAI Nouveaux themes de recherche dans les litteratures africaines, defis et possibilites d'ecrire et d'enseigner ces litteratures dans les langues africaines

ration, ne vont pas sans poser de sérieux problèmes qui ont jusqu'ici demeuré un point aveugle de la critique littéraire ou de l'anthropologie africaniste. Le premier et le plus massif de ce problème, parce qu'il est définitoire, est celui de la littérarité de ces nouveaux textes. L'exemple de Ngugi wa Thiong'o est à cet égard emblématique. Si ses nouveaux écrits en Gikuyu sont à coup sûr de nature politique, comment en établir ou en affirmer le statut littéraire, c'est-àdire esthétique ? Car n'est pas auteur de littérature qui veut, et de savoir écrire dans sa langue ne fait de personne un écrivain. Il faudrait pouvoir assurer que les écrits de Ngugi passent le test de litterarite (ce que les critiques russes ont nommé literaturnost). Ceci suppose l'existence d'une tradition littéraire crée au fil des générations par une communauté qui ait établi des critères de jugement esthétique et un seuil d'acceptabilité littéraire.

Il est fort possible qu'il y ait un "effet Mathieu" (Merton) lorsque Ngugi a décidé de passer de l'anglais au Gikuyu, sa réputation, obtenue grâce à ses écrits dans la langue de Shakespeare venant légitimer et ainsi établir son statut d'écrivain dans la langue africaine. Cette légitimation subreptice évacue l'incontournable problème de la littérarité de ses nouveaux écrits en Gikuyu, comme si ceci allait de soi. Or, chaque tradition littéraire ayant ses critères de littérarité, on ne saurait "transférer" ceux-ci d'une tradition à une autre.

Il se pose ainsi, à tout nouvel écrivain dans une nouvelle littérature écrite africaine un problème de compétence littéraire, et à chaque nouvelle littérature écrite africaine un problème de création d'une nouvelle tradition littéraire entée à coup sûr sur la littérarité de la littérature orale. Cette tradition sera l'œuvre d'une communauté de literati pétris dans l'influx littéraire oral de la langue africaine en question. (C'était le rôle dévolu à la communauté de connaisseurs de littérature dénommée THEORIA dans la tradition grecque).

Mais il se peut également, seul l'avenir nous le dira, que le seul fait, radicalement politique, d'écrire dans une langue africaine puisse constituer un élément ou un aspect de la littérarité. Après tout, disent certains critiques, le seul mérite de l'œuvre de Chaucer est d'exister en anglais, se posant comme il le fait en s'opposant aux œuvres en latin qui le précèdent.

Un autre défi et qui concerne les littératures en langues africaines de première génération (éthiopienne, zulu, swati, yoruba, kiswahili) est le développement d'un métalangage, d'une terminologie littéraire et d'un apparat critique appropriés. Nous dépendons trop des théories littéraires et 
África (São Paulo, 1978, Online), São Paulo, n. 42, p. 77-85, 2021

des méthodologies occidentales sans souvent nous poser la question de leur pertinence. Ici également, il y a beaucoup à apprendre de l'afflux africain. Le défi ici est à la fois épistémologique et méthodologique.

C'est le lieu de répéter qu'un travail de créativité en la matière exige l'articulation de l'afflux à l'influx. Nous abordons ainsi indirectement le vaste domaine de la traduction.

La tradition est pratiquement un continent négligé, une forêt vierge dans la pratique littéraire africaine. Il nous faut la prendre au sérieux, indiquer le rôle d'impulsion que peut jouer l'état (BERMAN, 2011). Le vaste programme de traduction devra comporter les traductions afro-africaines (zulu-yoruba; swahili-wolof... etc.) les traditions des classiques du monde entier dans les langues africaines (Popol Vuh, Upanishad, Cervantes, Goethe, Shakespeare... etc.) et vice-versa.

Expérimenter dans la tradition des œuvres orales et des essais critiques conduirait à un enrichissement terminologique, au renforcement de la pratique de la littérature comparée et au positionnement des littératures africaines dans un contexte de mondialisation.

Nos littératures en langues africaines ont devant elles un vaste horizon. Les ouvrir à la traduction, donc à d'autres littératures de par le monde élargirait cet horizon à l'infini.

Le passage à l'écriture s'est fait souvent avec l'adoption des genres et formes des littératures occidentales (roman, nouvelle, poésie versifiée, théâtre), des thématiques liées au contact avec l'Occident (tradition versus modernité) et aussi, il est bon de le noter pour pouvoir en faire la critique, un rétrécissement de l'horizon littéraire traditionnel du point de vue générique. Ainsi bien des genres ont été estompés parce que jugés typiques de la littérature orale mais "indignes" de l'écrit. Il en est ainsi notamment des formes fixes et gnomiques (proverbes, devinettes, devises... etc.) pourtant réputées pour leur raffinement stylistique donc chargées d'un potentiel élevé de créativité et de renouvellement littéraire.

Il se révèle ainsi que nos littératures orales avec leurs mythes, légendes, poésie divinatoire. Izibongo, zamu oriki, etc. constituent un gisement littéraire, un limon permanent insuffisamment exploité. La réappropriation du genre haiku, par la littérature japonaise nous indique le grand intérêt qu'il y a à se ressourcer dans les genres de nos traditions littéraires. 
OLABIYI YAI Nouveaux themes de recherche dans les litteratures africaines, defis et possibilites d'ecrire et d'enseigner ces litteratures dans les langues africaines

Une autre source d'extension de l'horizon thématique de nos littératures se trouve dans sa mise en dialogue avec les autres arts : musique, sculpture, peinture.

Dans le contexte traditionnel de l'oralité, ces arts sont souvent intégrés et se font écho au cours de rituels et autres cérémonies. Ainsi en yoruba, le poète oral est un 'sculpteur/orfèvre de la parole. Il n'y a pas de raison de ne pas continuer cette bonne tradition dans la littérature écrite. Et nous en avons, comme une invite, dans les poèmes de Carlos Drummond de Andrade. Ses poèmes sur les peintures, de Wega, Fayga Ostromer ou sur la musique de Beethoven sont des joyaux en la matière et donc dignes d'émulation.

Je ne peux terminer ce survol sans rapidement évoquer le rôle du Brésil et les attentes de l'élite littéraire africaine. Ce serait se méprendre sur les ruses de l'histoire que de penser que le Brésil n'a aucun rôle à jouer dans la renaissance des littératures africaines en langues africaines parce que ces langues ne sont plus parlées au Brésil, du moins dans la sphère publique. Et ce serait myopie que de confirmer la contribution du Brésil à la seule littérature lusophone africaine. Je pense que le Brésil pourrait et devrait jouer, un rôle d'éveilleur des consciences, rôle qu'il a déjà joué et continue de jouer dans la renaissance des religions traditionnelles africaines en Afrique grâce au flux et reflux du candomblé.

Il pourrait et devrait créer des chaires d'enseignement des langues et littératures africaines d'abord dans ses universités, puis à d'autres niveaux de l'éducation nationale brésilienne. Il pourrait et devrait aider les gouvernements africains comme les sociétés civiles africaines à l'indispensable technologisation des langues africaines, préalable à une appropriation massive de ces langues dès la petite enfance, créant les conditions de possibilité et de félicité d'une renaissance littéraire africaine à la hauteur d'une mondialisation juste et apaisée.

Il n'y a pas mieux qu'un poète, pour suggérer la voie/la voix de l'avenir. Et Carlos Drummond de Andrade, encore lui, nous la montrait dans les vers de ce poème dédié à son petit-fils Luis Mauricio, et qui méritent d'être mieux connus:

\author{
'É preciso \\ reinventar \\ Nagôs e Latinos
}


África (São Paulo, 1978, Online), São Paulo, n. 42, p. 77-85, 2021

\section{RefERENCES}

BERMAN, Antoine. Jacques Amyot, traducteur français. Essai sur les origines de la traduction en France. Paris: Belin, 2012. Collection: L'Extrême Contemporain.

CARNEIRO, Paulo de Berrêdo. Vers un nouvel humanisme. Paris: Éditions Seghers, 1971.

CESAIRE, Aimé. Moi, Laminaire. Paris: Seuil, 1991.

ANDRADE, Carlos Drummond de, (1978). As impurezas do branco. São Paulo: Companhia das Letras, 2012.

JASPERS, Karl, (1948). De l'Université. Lyon: Paragon/Vs, 2008.

OBENGA, Théophile. L'Université Africaine dans le cadre de l'Union Africaine. Paris: Pyramide Papyrus Press, 2003.

THIONG'O, Ngugi, wa. Moving the Center. London: James Currey, 2008. Series Studies in African Studies. 